\title{
FUNCTIONAL EVOLUTION EQUATIONS WITH NONCONVEX LOWER SEMICONTINUOUS MULTIVALUED PERTURBATIONS
}

\author{
A.G. IBRAHIM \\ Department of Mathematics \\ Faculty of Science \\ Cairo University \\ Giza, EGYPT
}

(Received August 28, 1996 and in revised form February 24, 1997)

\begin{abstract}
In this paper we prove some existence theorems concerning the solutions and integral solution for functional (delay) evolution equations with nonconvex lower semicontinuous multivalued perturbations
\end{abstract}

,KEY WORDS AND PHRASES: Functional evolution equations, $m$-accretive operators, integral solutions

1991 AMS SUBJECT CLASSIFICATION CODES: 34A60, 49J24

\section{INTRODUCTION}

Let $E$ be a Banach space, $r, T \in \mathbb{R}^{+}$and $I=[a, b]$ Let us denote

$C_{E}([-r, T])$ the vector space of all continuous functions from $[-r, T]$ to $E$ endowed with the uniform topology

For all $t \geq 0, s_{t}: C_{E}([-r, t]) \rightarrow C_{E}([-r, 0])$,

$$
\left(s_{t} f\right)(\theta)=f(t+\theta), \quad \forall \theta \in[-r, 0] .
$$

$A: I \times E \rightarrow 2^{E}$ such that $A(t,$.$) is an m-accretive multivalued operator$ $P_{w c}(E)$ the family of nonempty weakly compact subsets of $E$ In this paper we are concerned with the following problems

(1) Existence of solutions of the perturbated evolution equation with delay

$$
(P) \begin{cases}u^{\prime}(t) \epsilon-A(t, u(t))+F\left(t, s_{t} u\right) & \text { a e on } I, \\ u \equiv \psi & \text { on }[-r, 0]\end{cases}
$$

where $F: I \times C_{E}([-r, 0]) \rightarrow P_{w c}(E)$ is a multivalued function such that $F(t,$.$) is lower$ semicontinuous and $\psi \in C_{E}([-r, 0])$ is arbitrary but fixed.

(2) Existence of solutions of the perturbated evolution equation with delay

$$
(Q) \begin{cases}u^{\prime}(t) \epsilon-N_{\Gamma(t)}(u(t))+F\left(t, s_{t} u\right) & \text { a e on } I \\ u \equiv \psi & \text { on }[-r, 0]\end{cases}
$$

where $N_{\Gamma(t)}(x)$ is the normal cone of the convex set $\Gamma(t)$ at the point $x \in E ; t \in I$ It should be noticed that the problem $(Q)$ is not a special case of the problem $(P)$

(3) Existence of integral solutions of $(P)$, when the operator $A$ is independent of $t$, under conditions that are weaker than those imposed in $(P)$

The results obtained in the present paper generalized the following interesting known cases

Problem $(P)$ for which the dual of $E$ is uniformly convex, $A(t,$.$) is an m-accretive single-valued$ operator and $F$ is a Lipschitz single-valued function cf Kartsatos and Parrott [1] 
Problem $(P)$ for which $E$ is reflexive, $A(t,$.$) is an m-accretive multivalued operator and F$ is a Lipschitz single-valued function cf Tanaka [2]

Problems $(P)$ and $(Q)$ without delay cf Cichon [3], [4], Ibrahim [5] and the references therein

\section{NOTATIONS AND DEFINITIONS}

Let $E^{*}$ be the dual of $E, E_{\sigma}$ the Banach space $E$ endowed with the weak topology $\sigma\left(E, E^{*}\right)$ If $B$ is a multivalued operator from $E$ to $2^{E}$ then $B$ is said to be accretive if for each $\lambda>0, x_{1}, x_{2} \in D(B)$ (the domain of $B$ ), $y_{1} \in B\left(x_{1}\right)$ and $y_{2} \in B\left(x_{2}\right)$ we have

$$
\left\|x_{1}-x_{2}\right\| \leq\left\|x_{1}-x_{2}+\lambda\left(y_{1}-y_{2}\right)\right\| .
$$

We say that $B$ is m-accretive if $B$ is accretive and if there exists $\lambda>0$ such that $R(I+\lambda B)=E$, where $I$ is the identity map It is known that if $B$ is m-accretive, then for every $\lambda>0$ the resolvant $J_{\lambda} B=(I+\lambda B)^{-1}$ and the Yosida approximation of $B ; B_{\lambda}=\left(I-J_{\lambda} B\right) / \lambda$, are defined everywhere The generalized domain of $B$ is defined by

$$
D^{*}(B)=\left\{x \in E:|B(x)|=\lim _{\lambda \rightarrow \infty}\left\|B_{\lambda} x\right\|<\infty\right\} .
$$

For the properties of $m$-accretive multivalued operators refer to [6] and [7]

If $C$ is a convex subset of $E$ and $x \in C$, then the normal cone of $C$ at $x$ is defined by

$$
N_{C}(x)=\left\{y \in E^{*}:\langle y, z-x\rangle \leq 0, \forall z \in C\right\} .
$$

Now we recall some concepts concerning multivalued functions Let $Y$ be a locally convex space and let $G: E \rightarrow 2^{Y}-\{\phi\} \quad$ We say that $G$ is lower semicontinuous (resp. upper semicontinuous) if for every open $V$ in $Y$ the set $\{x \in E: G(x) \cap V \neq \phi\}$ (resp $\{x \in E: G(x) \subset V\}$ ) is open in $E$. We say that $G$ is lower semicontinuous (resp. upper semicontinuous) in the Kuratowski sense iff for all $v_{n} \rightarrow v$ in $E$, $G(v) \subseteq \lim _{n \rightarrow \infty} \inf G\left(v_{n}\right)\left(\operatorname{resp} \lim _{n \rightarrow \infty} \sup G\left(v_{n}\right) \subseteq \mathrm{G}(v)\right)$, where

$$
\begin{aligned}
& \lim _{n \rightarrow \infty} \inf G\left(v_{n}\right)=\left\{z \in Y: z=\lim _{n \rightarrow \infty} z_{n}, z_{n} \in G\left(v_{n}\right), \forall n \geq 1\right\}, \\
& \lim _{n \rightarrow \infty} \sup G\left(v_{n}\right)=\left\{z \in Y: z=\lim _{n \rightarrow \infty} z_{n_{k}}, z_{n_{k}} \in G\left(v_{n_{k}}\right), \forall k \geq 1\right\} .
\end{aligned}
$$

If $E$ is metrizable then lower semicontinuity and lower semicontinuity in the Kuratowski sense are equivalent (cf [8], [9])

The following known result will be used in the sequel

LEMMA 2.1 [6]. For every $t \in I$, let $A(t,$.$) be an m-accretive multivalued operator from E$ to $2^{E}-\{\phi\}$ satisfying the following condition:

$\left(C_{1}\right)$ There exist $\lambda_{0}>0$, a continuous function $h: I \rightarrow E$ and a nondecreasing continuous function $L:[0, \infty) \rightarrow[0, \infty)$ such that for all $\lambda \in\left(0, \lambda_{0}\right)$ and for almost $t, s \in I$,

$$
\left\|A_{\lambda}(t, x)-A_{\lambda}(s, x)\right\| \leq\|h(s)-h(t)\| L(\|x\|), \quad \forall x \in E .
$$

Then $D^{*}(A(t,)$.$) and \bar{D}(A(t,)$.$) are independent of t$

So if $A$ is as in Lemma 2.1 we may write $D^{*}(A):=D^{*}(A(t,)$.$) and \overline{D(A)}:=\overline{D(A(t, .))} ; t \in I$ respectively

LEMMA 2.2 [10]. Let $E$ be a Banach space and $M$ a compact metric space If $T$ is a lower semicontinuous multivalued function on $M$ and with nonempty closed decomposable values in $L_{E}^{1}(I)$, then $T$ has a continuous selection.

\section{EXISTENCE OF SOLUTIONS FOR THE PROBLEMS $(P)$ AND $(Q)$}

To prove our results we need the following lemmas

LEMMA 3.1. Let $\psi$ be an element of $C_{E}([-r, 0])$ and $\beta$ be a positive real number The set 


$$
\chi=\left\{u \in C_{E}([-r, 0]): u \equiv \psi \text { on }[-r, 0] \text { and } u(t)=\psi(0)+\int_{0}^{t} f(s) d s ; f \in K_{\beta}\right\}
$$

is nonempty and convex, where $K_{\beta}=\left\{f \in L_{E}^{1}(I):|f(t)| \leq \beta\right.$ a e on $\left.I\right\} \quad$ If $E$ is reflexive then $\chi$ is compact subset of $C_{E_{o}}([-r, T])$ If, in addition, $E$ is separable then $\chi$ is metrizable

PROOF. It is obvious that $\chi$ is nonempty, convex and equicontinuous and that the set $\{u(t): u \in \chi\} ; t \in I$, is bounded So, if $E$ is reflexive then, $\chi$ is relatively compact in $C_{E_{\sigma}}([-r, T])$ by Ascoli's theorem Let us verify that $\chi$ is closed in $C_{E_{\sigma}}([-r, T])$ Let $\left(u_{n}\right)$ be a sequence in $\chi$ converging to $u \in C_{E_{o}}([-r, T]) \quad$ Then $u \equiv \psi$ on $[-r, 0]$ and for each $n \geq 1$ there exists $f_{n} \in K_{\beta}$ such that $u_{n}(t)=\psi(0)+\int_{0}^{t} f_{n}(s) d s ; t \in I \quad$ Since $E$ is reflexive, $K_{\beta}$ is weakly compact in $L_{E}^{1}(I)$ Hence, the sequence $\left(f_{n}\right)$ has a subsequence, denoted again by $\left(f_{n}\right)$, converging weakly to $f \in K_{\beta}$ Then $u(t)=\psi(0)+\int_{0}^{t} f(s) d s ; t \in I \quad$ This proves that $\chi$ is closed in $C_{E_{o}}([-r, T])$ Now if $E$ is separable then so is $L_{E}^{1}(I)$ Consequently, $K_{\beta}$ is metrizable Since $\chi$ is isomorphic to $\{\psi(0)\} \times K_{\beta}$, then $\chi$ is metrizable

LEMMA 3.2. Let $G$ be a multivalued function from $E_{\sigma}$ to the nonempty closed subsets of $E$ such that $G$ is lower semicontinuous in the Kuratowski sense. If $\left(x_{n}\right)$ is a sequence converging to $x$ in $E_{\sigma}$, then for every $z \in E$,

$$
\lim _{n \rightarrow \infty} \sup d\left(z, G\left(x_{n}\right)\right) \leq d\left(z, \lim _{n \rightarrow \infty} \inf G\left(x_{n}\right)\right) \leq d(z, G(x)) .
$$

PROOF. Let $y \in \lim _{n \rightarrow \infty} \inf G\left(x_{n}\right)$ Then there exists a sequence $\left(y_{n}\right)$ such that $y_{n} \in G\left(x_{n}\right) ; n \geq 1$ and $y_{n} \rightarrow y$ as $n \rightarrow \infty$ For any $z \in E$ we have

$$
\lim _{n \rightarrow \infty} \sup d\left(z, G\left(x_{n}\right)\right) \leq \lim _{n \rightarrow \infty} \sup \left\|z-y_{n}\right\|=\|z-y\|
$$

which proves the first inequality The second inequality follows from the lower semicontinuity of $G$

THEOREM 3.1. Let $E$ be a reflexive separable Banach space Let $A(t,.) ; t \in I$ be an m-accretive multivalued operator from $E$ to $2^{E}-\{\phi\}$ satisfying condition $\left(C_{1}\right)$ together with the following conditions

$\left(C_{2}\right)$ There exist $\mu>0$ such that for all $x \in E$, the function $w_{x}: t \rightarrow(I+\mu A(t, .))^{-1}$ belongs to $L_{E}^{2}(I)$

$\left(C_{3}\right)$ For all $r>0$ there exists $\delta(r)>0$ such that for all $\lambda>0$ and all $x \in \bar{D}(A)$ with $\|x\|<r$,

$$
\left\|J_{\lambda} A(0, x)-x\right\| \leq \lambda \delta(r)
$$

Let $F$ be a measurable multivalued function from $I \times C_{E}([-r, 0])$ to $P_{w c}(E)$ satisfying the following conditions

$\left(F_{1}\right)$ There exists $\alpha>0$ such that

$$
\sup \{\|y\|: y \in F(t, u)\} \leq \alpha, \quad \forall(t, u) \in I \times C_{E}([-r, 0]) .
$$

$\left(F_{2}\right)$ For all $t \in I, F(t,$.$) is lower semicontinuous in the sense of Kuratowski from C_{E_{\sigma}}([-r, 0])$ to E

$\left(F_{3}\right)$ For all $u \in C_{E}([-r, 0])$ the multivalued function $t \rightarrow F\left(t, s_{t} u\right)$ admits a measurable selection Then for every $\psi \in C_{E}([-r, 0])$ with $\psi(0) \in D^{*}(A)$, the problem $(P)$ has a solution.

PROOF. We split the proof into the following three steps

(1) Let $f \in K_{\alpha}=\left\{g \in L_{E}^{1}(I):\|g(t)\| \leq \alpha\right.$ a.e on $\left.I\right\}$. Since $A$ satisfies conditions $\left(C_{1}\right),\left(C_{2}\right)$ and $\left(C_{3}\right)$, then by Theorem 4 of [5], there exists a unique absolutely continuous function $u_{f}: I \rightarrow E$ such that

(i) $u_{f}^{\prime}(t) \in-A(t, u(t))+f(t)$ a e. on $I, u_{f}(0)=\psi(0)$,

(ii) $\left\|u_{f}(t)\right\| \leq \beta_{1}=(\alpha+1) T+L(r) \sup _{t \in I}\|h(t)\|+\delta(r), \forall t \in I$, where $r=\alpha(1+L(\|\psi(0)\|))+\left|A\left(0, x_{0}\right)\right|$, 
(iii) the function $f \rightarrow u_{f}$ is continuous from $K_{\alpha}$ to $C_{E_{o}}(I)$

(2) Set $\chi_{1}=\left\{u \in C_{E}([-r, T]), u \equiv \psi\right.$ on $[-r, 0]$ and $\left.u(t)=\psi(0)+\int_{0}^{t} f(s) d s, f \in K_{\beta}\right\} \quad$ By Lemma $31, \chi_{1}$ is a compact subset of $C_{\sigma}([-r, T])$ and is metrizable. Define a multivalued function $T_{1}$ on $\chi_{1}$ by $T_{1}(u)=\left\{f \in K_{\alpha}: f(t) \in F\left(t, s_{t} u\right)\right.$ a e on $\left.I\right\} \quad$ In this step we prove that $T_{1}$ has a continuous selection $V_{1}: \chi_{1} \rightarrow K_{\alpha} \quad$ For this purpose, we show that $T_{1}$ satisfies the conditions of Lemma 22 Condition $\left(F_{3}\right)$ assures that the values of $T_{1}$ are nonempty Moreover, if $D$ is a measurable subset of $I$ and $g_{1}, g_{2} \in T_{1}(u)$ for some $u \in \chi_{1}$, then the function $g=N_{D} g_{1}+N_{I-D} g_{2}$ belongs to $T_{1}(u)$, where $N$ is the characteristic function. Then the values of $T_{1}$ are decomposable It remains to prove that $T_{1}$ is lower semicontinuous Since $\chi_{1}$ is compact metrizable in $C_{E \sigma}([-r, T])$, it suffices to show that $T_{1}$ is lower semicontinuous in the Kuratowski sense So, let $\left(u_{n}\right)$ be a sequence in $\chi_{1}$ converging to $u \in \chi_{1}$, with respect to the topology on $C_{E \sigma}([-r, T])$ and let $g \in T_{1}(u)$ Since $F$ is measurable, then for all $n \geq 1$ the multivalued function

$$
t \rightarrow B_{n}(t)=\left\{z \in F\left(t, s_{t} u_{n}\right):\|g(t)-z\|=d\left(g(t), F\left(t, s_{t} u_{n}\right)\right)\right\}
$$

has a measurable selection $g_{n}: I \rightarrow E$. Thus, by Lemma 32 , for all $t \in I$,

$$
\begin{aligned}
\lim _{n \rightarrow \infty}\left\|g(t)-g\left(t_{n}\right)\right\| & \leq \lim _{n \rightarrow \infty} \sup d\left(g(t), F\left(t, s_{t} u_{n}\right)\right) \\
& \leq d\left(g(t), \lim _{n \rightarrow \infty} \inf F\left(t, s_{t} u_{n}\right)\right) \\
& =d\left(g(t), F\left(t, s_{t} u\right)\right)=0 .
\end{aligned}
$$

This means that $T_{1}$ is lower semicontinuous and hence there exists a continuous function $V_{1}: \chi_{1} \rightarrow K_{a}$ such that $V_{1}(x) \in T(x), \forall x \in \chi_{1}$

(3) Define a function $\theta: \chi_{1} \rightarrow \chi_{1}$ by $\theta(x)=u_{f}, f=V_{1}(x)$ By (iii) of the first step, $\theta$ is continuous Hence, by Tichonoff's fixed point theorem, there exists $u \in \chi_{1}$ such that $u=u_{f}, f=V_{1}(u) \in T_{1}(u)$ This means that $u^{\prime}(t) \in-A(t, u(t))+f(t)$ and $f(t) \in F\left(t, s_{t} u\right)$ a e on $I$ The theorem is thus proved.

THEOREM 3.2. Let $H$ be a Hilbert space and $F$ be a measurable multivalued function from $I \times C_{H}([-r, 0])$ to $P_{w c}(H)$ satisfying conditions $\left(F_{1}\right),\left(F_{2}\right)$ and $\left(F_{3}\right)$ Let $\Gamma$ be a multivalued function from $I$ to the family of nonempty closed convex subsets of $H$, with compact graph $G$ and satisfies the following conditions.

$\left(\Gamma_{1}\right)$ There exists $\gamma>0$ such that $\left\|x-\operatorname{proj}_{\Gamma(t)} x\right\| \leq \gamma(\tau-t)$ for all $(t, x) \in G$ and all $\tau \in I,(t<\tau)$

$\left(\Gamma_{2}\right)$ The function $(t, x) \rightarrow \delta^{x}(x, \Gamma(t))=\sup \{(x, y): y \in \Gamma(t)\}$ is lower semicontinuous on $I \times B_{\sigma}$, where $B_{\sigma}$ is the relative weak topology

Then for all $\psi \in C_{E}([-r, 0])$ with $\psi(0) \in \Gamma(0)$, the problem $(Q)$ has a solution

PROOF. We split the proof into the following three steps

(1) Let $f \in K_{\alpha} \quad$ Since $\Gamma$ has a compact graph and satisfies conditions $\left(\Gamma_{1}\right)$ and $\left(\Gamma_{2}\right)$ then by Theorem 31 [11], there exists a unique absolutely continuous function $u_{f}: I \rightarrow H$ such that

(i) $u_{f}^{\prime}(t) \in-N_{\Gamma(t)}(u(t))+f(t)$ a.e. on $I$,

(ii) $u_{f}(0)=\psi(0), u_{f}(t) \in \Gamma(t), \forall t \in I$,

(iii) $\left\|u_{f}(t)\right\| \leq \beta_{2}=T(\gamma+\alpha), \forall t \in I$ and the function $f \rightarrow u_{f}$ is continuous from $K_{\alpha}$ to $C_{H_{\sigma}}$

(2) Set $\chi_{2}=\left\{u \in C_{H}([-r, T]): u=\psi\right.$ on $[-r, 0]$ and $\left.u(t)=\psi(0)+\int_{0}^{t} f(s) d s, f \in K_{\beta_{2}}\right\}$ and define a multivalued function $T_{2}$ on $\chi_{2}$ by $T_{2}(u)=\left\{f \in K_{\alpha}: f(t) \in F\left(t, s_{t} u\right)\right.$ a.e on $\left.I\right\}$ As in the second step of the proof of Theorem 3.1 we can show that $T_{2}$ has a continuous selection $V_{2}: \chi_{2} \rightarrow K_{\alpha}$

(3) Define the function $\theta: \chi_{2} \rightarrow \chi_{2}$ by $\theta(x)=u_{f}, f=V_{2}(x)$ As in the third step of the proof of Theorem 3.1, we can show that there exists a unique $u \in \chi_{2}$ such that $u=u_{f}, f \in T_{2}(u)$ Clearly $u$ is a solution of $(Q)$ 


\section{EXISTENCE OF INTEGRAL SOLUTIONS FOR THE PROBLEM $(P)$ WHEN THE OPERATOR $A$ IS INDEPENDENT OF TIME}

In this section $A$ denotes a multivalued operator from $E$ to $2^{E}-\{\phi\} \quad$ Consider the evolution equation

$$
\left(P^{*}\right)\left\{\begin{array}{l}
u^{\prime}(t) \in-A(u(t))+f(t) \text { a e on } I \\
u(0)=x_{0} \in \overline{D(A)}
\end{array}\right.
$$

where $f \in L_{E}^{1}(I) \quad$ By an integral solution of $\left(P^{*}\right)$ we mean a continuous function $u: I \rightarrow \overline{D(A)}$ with $u(0)=x_{0}$ such that

$$
\|u(t)-z\| \leq\|u(s)-z\|+\int_{s}^{t}[u(r)-z, f(r)-y]_{+} d r
$$

for each $z \in D(A), y \in A(z)$ and $0 \leq s \leq t<T$, where

$$
\left[x_{1}, x_{2}\right]_{+}=\lim _{h \downarrow 0}\left(\left\|x_{1}+h x_{2}\right\|-\left\|x_{1}\right\|\right) / h, \forall x_{1}, x_{2} \in E .
$$

It is known that [7] if $A$ is an m-accretive operator then for each $\left(x_{0}, f\right) \in \overline{D(A)} \times L_{E}^{1}(I)$, the problem $\left(P^{*}\right)$ has a unique integral solution $u_{f}$, such that the function $f \rightarrow u_{f}$ is continuous In this section we are concerned with the existence of integral solutions of the functional evolution equation

$$
\left(P^{* *}\right) \begin{cases}u^{\prime}(t) \in-A(u(t))+F\left(t, s_{t} u\right) & \text { a e on } I \\ u \equiv \psi & \text { on }[-r, 0]\end{cases}
$$

where $F$ is a multivalued function from $I \times C_{E}([-r, 0])$ to $2^{E}-\{\phi\}, S_{t} ; t>0$ is the operator of translation defined in section 1 and $\psi$ is a given function, belongs to $C_{E}([-r, 0])$ with $\psi(0) \in \overline{D(A)}$ By an integral solution of $\left(P^{* *}\right)$ we mean a continuous function $u:[-r, T] \rightarrow E$ with $u \equiv \psi$ on $[-r]$,0 , such that $u$ is an integral solution of the evolution equation $u^{\prime}(t) \in-A(u(t))+f(t), u(0)=\psi(0)$, where $f \in L_{E}^{1}(I)$ and $f(t) \in F\left(t, s_{t} u\right)$, a e on $I$

We say that the operator $A: E \rightarrow 2^{E}-\{\phi\}$ has the (M)-property ([7], [12]) if for each $x_{0} \in D(A)$ and each uniformly integrable subset $Q$ of $L_{E}^{1}(I)$, the set $\left\{u_{g}: g \in Q\right\}$ is a relatively compact subset of $C_{E}(I)$ where $u_{g}$ is the unique integral solution of the evolution equation $u^{\prime}(t) \in-A(u(t))+g(t)$ a $\mathrm{e}$ on $I ; u(0)=x_{0}$. It is well known that ([7], [12]) if the proper operator $-A$ generates a compact semigroup (via Crandall-Liggett's exponential formula [3], [13]), then $A$ has the property (M)

THEOREM 4.1. Let $E$ be a Banach space and $A$ an m-accretive multivalued operator from $E$ to $2^{E}-\{\phi\}$ having the (M)-property. Let $F$ be a measurable multivalued function from $I \times C_{E}([-r, 0])$ to the non-empty closed subsets of $E$ satisfying the condition $\left(F_{3}\right)$ together with the following conditions

$\left(F_{4}\right)$ There exists a function $h \in L_{\mathbb{R}}^{1}(I)$ such that

$$
\sup \{\|z\|: z \in F(t, u)\} \leq h(t), \quad \forall(t, u) \in I \times C_{E}([-r, 0]) .
$$

$\left(F_{5}\right)$ For all $t \in I, F(t,):. C_{E}([-r, 0]) \rightarrow E$ is lower semicontinuous in the Kuratowski sense Then for all $\psi \in C_{E}([-r, 0])$ with $\psi(0) \in \overline{D(A)}$, the problem $\left(P^{* *}\right)$ has an integral solution

PROOF. Consider the set $Q=\left\{f \in L_{E}^{1}(I):\|f(t)\| \leq h(t)\right.$ a e. on $\left.I\right\} \quad$ One can easily show that $Q$ is nonempty and uniformly integrable subset of $L_{E}^{1}(I)$ As mentioned above, for each $f \in Q$ there exists a unique continuous function $u_{f}: I \rightarrow \overline{D(A)}$ such that $u_{f}$ is the unique integral solution of the evolution equation $u^{\prime}(t) \in A(u(t))+f(t), u(0)=\psi(0)$ and the function $f \mapsto u_{f}$ is continuous from $Q$ to $C_{E}(I)$. Let $\chi^{*}=\overline{\left\{u_{f}^{*} \in C_{E}([-r, T]): f \in Q\right\}}$, where $u_{f}^{*} \equiv \psi$ on $[-r, 0]$ and $u_{f}^{*} \equiv u_{f}$ on $I$ Since $a$ has the property $(\mathrm{M}), \chi^{*}$ is compact in the metric space $C_{E}([-r, T])$ Now, define a multivalued function $T$ on $\chi^{*}$ by $T(x)=\left\{f \in L_{E}^{1}(I): f(t) \in F\left(t, s_{t} x\right)\right.$ a e on $\left.I\right\} \quad$ As in the second step of the proof of Theorem 31 , we can show that $T$ has a continuous selection $V: \chi^{*} \rightarrow L_{E}^{1}(I)$ 
Also, define a function $\Phi: \chi^{*} \rightarrow \chi^{*}, \Phi(x)=u_{f}^{*}, f=V(x)$ The function $\Phi$ is clearly continuous and hence has a fixed point $x \in \chi^{*}$ It is obvious that $x$ is the desired solution

\section{EXAMPLES}

In this section we give some examples illustrating the scope of the results developed in sections 3 and 4

EXAMPLE 1. Let for all $t \in I, A(t)=B-h(t)$ where $h: I \rightarrow E$ is integrable and $B$ is an maccretive operator on $E$ Clearly $A(t)$ is $\mathrm{m}$-accretive for all $t \in I$ Let $\lambda>0, s, t \in I$ and $x \in E$ Then

$$
\left\|A_{\lambda}(t, x)-A_{\lambda}(s, x)\right\| \leq \frac{1}{\lambda}\left\|J_{\lambda} A(t, x)-J_{\lambda} A(s, x)\right\| \leq\|h(t)-h(s)\| \text {. }
$$

Hence condition $\left(C_{1}\right)$ of Lemma 2.1 holds

EXAMPLE 2. In [6] there are several examples for operators $A$ such that for every $t \in I, A(t)$ is m-accretive and satisfies condition $\left(C_{1}\right)$

EXAMPLE 3. Let $H$ be a real Hilbert space with inner product $(.,$.$) and let \Phi: H \rightarrow H$ be a proper lower semicontinuous convex function. The set $\partial \Phi(x)=\{z \in H: \Phi(x) \leq \Phi(y)+\langle x-y, z\rangle$ for each $y \in H\}$ is called the subdifferential of $\Phi$ at the point $x$ We recall that $D(\partial \Phi)=$ $\left\{x \in H: \partial \Phi(x)\right.$ is nonempty\}. Now if we define an operator $A: D(A)=D \partial(\Phi) \rightarrow 2^{H}$ by $A(x)=\partial \Phi(x)$, then $A$ is m-accretive and the following conditions are equivalent [7]

(i) For each $\lambda>0$, the resolvent $J_{\lambda} A$ is a compact operator

(ii) The function $\Phi$ is of compact type

(iii) The semigroup generated by the operator $-A$ is compact

EXAMPLE 4. Take $E=L_{\mathbb{R}}^{2}([0, \pi])$ and let us define $A: D(A) \subseteq E \rightarrow E$ by $A u=-u^{(2)}(t)$ for each $u \in D(A)$ where $D(a)=\left\{u \in E: u^{(2)} \in E, u(0)=u(\pi)=0\right\} \quad$ The operator $A$ is m-accretive and the semigroup $\{S(t): t>0\}$ generated by $-A\left(S(t)=\lim _{n \rightarrow \infty}\left(I+\frac{t}{n} A\right)^{-n}\right)$ is compact [7]

\section{REFERENCES}

[1] KARTSATOS, A.G. and PARROTT, M.E, A method of lines for a nonlinear abstract functional evolution equation, Trans. Amer. Math. Soc. 286 (1984), 73-89.

[2] TANAKA, N, On the existence of solutions of functional evolution equations, Nonlinear Analysis Methods and Applications 12(10) (1988), 1087-1104

[3] CICHON, M., Multivalued perturbations of m-accretive differential inclusions in a non-separable Banach space, Annales Societatis Math. Polonae. Ser. 1 XXXII (1992).

[4] CICHON, M., Non compact perturbations of m-accretive operators in general Banach spaces, Comment Math. Univ. Carolinae 333 (1992), 403-409.

[5] IBRAHIM, A G, Lower semicontinuous non-convex perturbations of m-accretive differential inclusions in Banach spaces, Periodice Mathematice Hungarica 27(1) (1993), 1-15

[6] EVANS, L C., Nonlinear evolution equations in an arbitrary Banach space, Israel Journal Math. 26 (1977), 1-42.

[7] VRABIE, I.L, Compactness methods for nonlinear evolutions, Pttman Monographs Surveys Pure Appl. Math. 32, Longman Sci Tech Harlow (1987)

[8] DELAHAYE, T.P. and DENEL, T., The continuities of the point to set map definitions and equivalences, Math. Programming Study 10 (1972), 57-94.

[9] MOSCO, U., Convergence of convex sets and solutions of variational inequalities, Adv. in Math. 3 (1969), 510-583.

[10] FRYSZKOWSKI, A., Continuous selections for a class of non-convex multivalued maps, Studia Math. 76 (1983), 163-174.

[11] IBRAHIM, A.G, Non-convex lower semicontinuous perturbations of an evolution problem in Hilbert spaces, Bull. Fac. Sci. Assutt. Univ. Egypt 22(2-C) (1993), 13-28.

[12] MITIDIERI, E and VRABIE, I.I., Differential inclusions governed by non-convex perturbations of m-accretive operators, Differential Integral Equations 2 (1989), 525-531

[13] CRANDALL, M.G., Generations of semigroups of nonlinear transformations on general Banach spaces, Amer. J. Math. 93 (1971), 265-298 


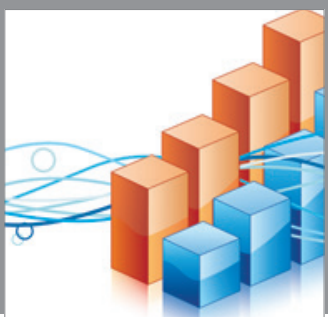

Advances in

Operations Research

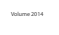

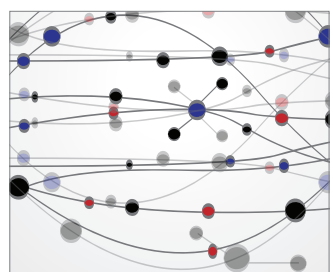

\section{The Scientific} World Journal
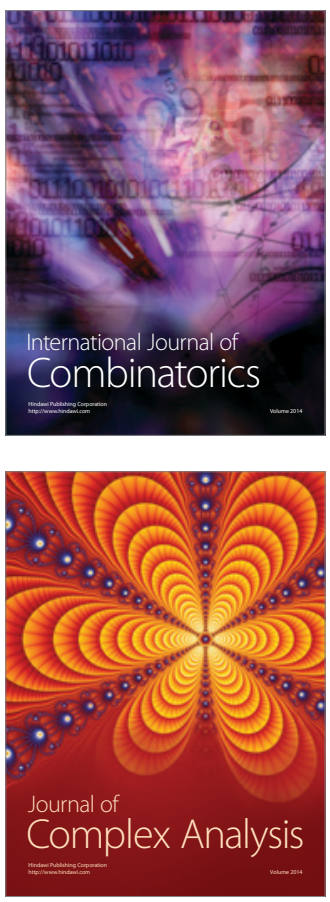

International Journal of

Mathematics and

Mathematical

Sciences
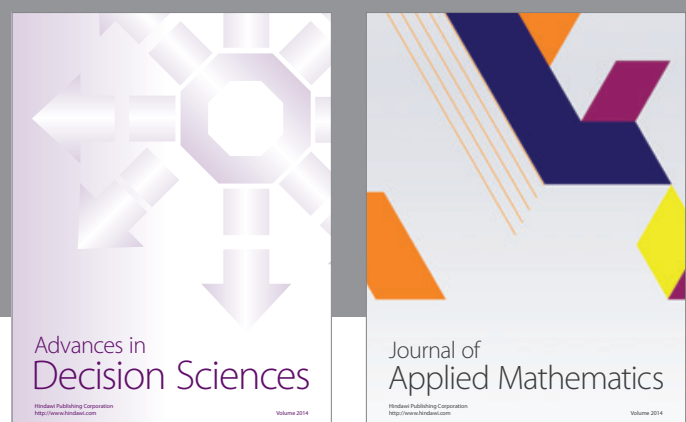

Journal of

Applied Mathematics
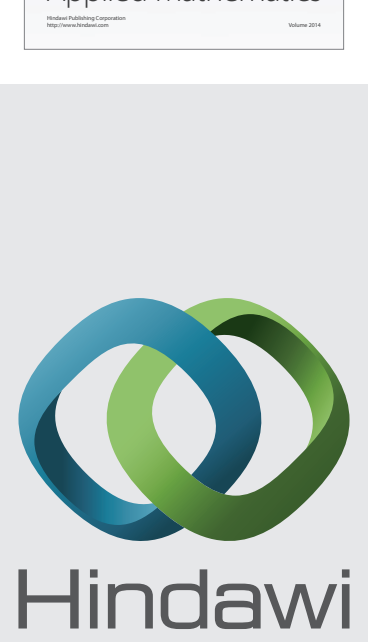

Submit your manuscripts at http://www.hindawi.com
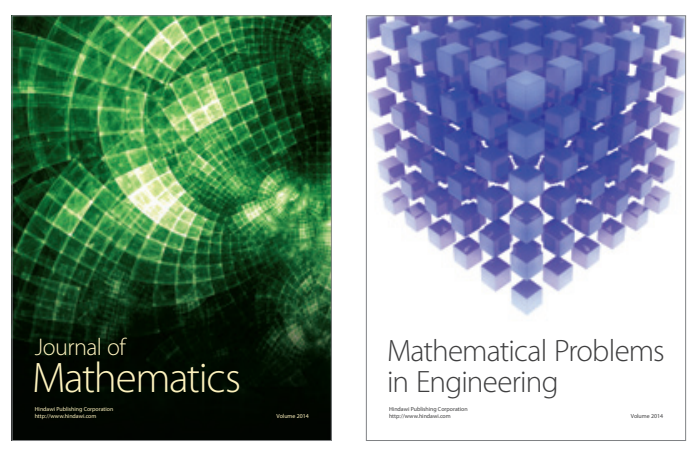

Mathematical Problems in Engineering
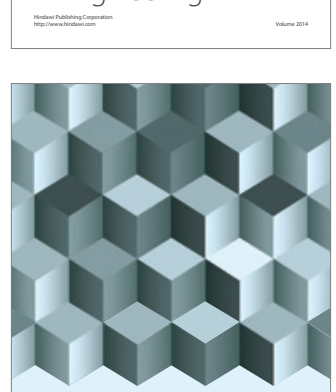

Journal of

Function Spaces
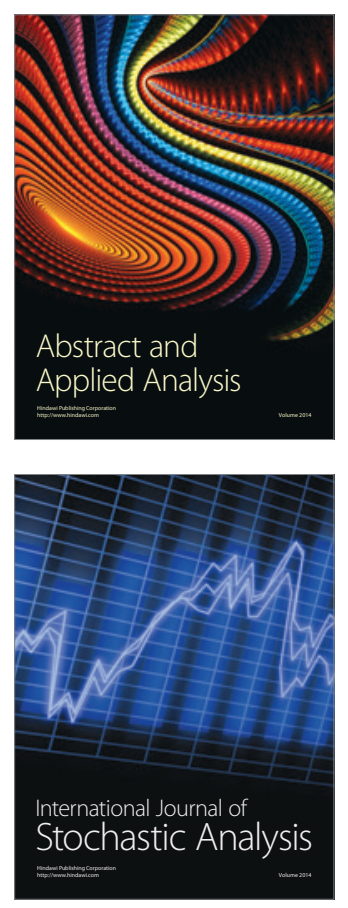

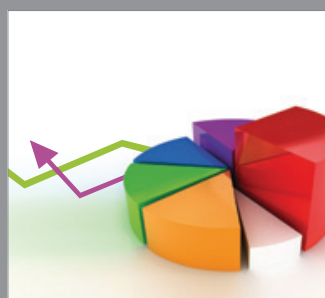

ournal of

Probability and Statistics

Promensencen
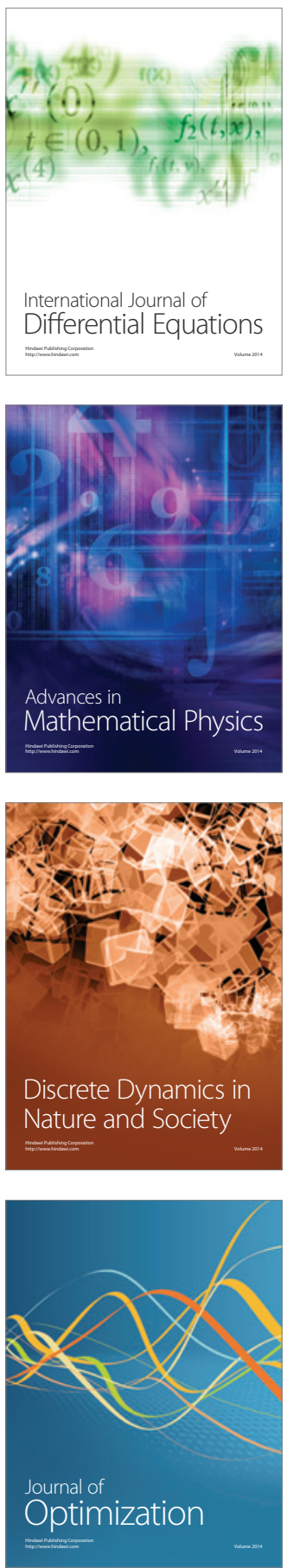